\title{
Classification of Boar Spermatozoid Head Images Using a Model Intracellular Density Distribution
}

\author{
Lidia Sánchez ${ }^{1}$, Nicolai Petkov ${ }^{2}$, and Enrique Alegre ${ }^{1}$ \\ ${ }^{1}$ Department of Electrical and Electronics Engineering, \\ University of León, Campus de Vegazana s/n, 24071 León, Spain \\ ${ }^{2}$ Institute of Mathematics and Computing Science, \\ University of Groningen, P.O. Box 800, 9700 AV Groningen, The Netherlands \\ \{lidia, enrique.alegre\}@unileon.es, petkov@cs.rug.nl
}

\begin{abstract}
We propose a novel classification method to identify boar spermatozoid heads which present an intracellular intensity distribution similar to a model. From semen sample images, head images are isolated and normalized. We define a model intensity distribution averaging a set of head images assumed as normal by veterinary experts. Two training sets are also formed: one with images that are similar to the model and another with non-normal head images according to experts. Deviations from the model are computed for each set, obtaining low values for normal heads and higher values for assumed as non-normal heads. There is also an overlapped area. The decision criterion is determined to minimize the sum of the obtained false rejected and false acceptance errors. Experiments with a test set of normal and non-normal head images give a global error of $20.40 \%$. The false rejection and the false acceptance rates are $13.68 \%$ and $6.72 \%$ respectively.
\end{abstract}

\section{Introduction}

Semen quality assessment is an important subject in fertility studies: semen analysis is a keystone in the clinical workup of infertile male patients and semen assessment is a critical stage in artificial insemination processes carried out in veterinary medicine. Pig and cattle farmers regularly acquire semen for artificial insemination from national and international breeding companies whose main objective is to generate and supply semen from boars and bulls of high genetic value. These companies are aware that they must maintain high standards of product, and therefore subject the semen to rigorous quality control procedures. Some of them use computerised methods for sperm evaluation, thus obtaining information about the quality of overall motility and morphology, and others couple this with tests to evaluate sperm plasma membrane and acrosomal integrity. A precise prediction of fertility cannot be provided, although problematic samples can usually be distinguished.

Whereas the majority of currently applied methods for inspection of animal gametes were developed for the analysis of human semen morphology and subsequently adapted for semen of other species, there is a continuing development of new methodologies [12]. Such improvements have increased the sensitivity of automated analysis, allowing the recognition of minuscule differences between sperm cells. However, experts do not know a lot about the influence of these morphological alterations in male 
fertility [34]. Several authors have proposed different approaches to classify subpopulations or to describe shape abnormalities using image processing techniques. Most of them use CASA (Computer Aided Sperm Analysis) systems [5]6] or propose new description and classification methods [7/8 9]10|11].

Although acrosome integrity and plasma membrane integrity determine the sperm viability because their enzymes take part in the oocyte penetration process, some possible features obtained of density distribution or intracellular texture are not considered. It is a visually observable fact that spermatozoid heads present a variety of cellular textures and the experts know that they are determined by their corresponding cytoplasmic densities. Our research is focused on finding a correlation between certain patterns of intracellular density distribution and semen fertility.

In this approach, veterinary experts first assume that a certain intracellular density distribution is characteristic of healthy cells. Then a distribution model for normal heads is obtained. Traditional techniques such as vital and fluorescent stains are used to assess the sperm capacitation of a sample, and experts try to find a correlation between the above mentioned classification and semen fertility. The aim of this research is to define a pattern of intracellular density distribution that corresponds to semen fertility, as determined by traditional techniques. This approach can lead to the use of digital image processing for sperm fertility estimation instead of expensive staining techniques.

In the current work, we analyse grey-level images of boar spermatozoid heads comprised in boar semen samples, Fig. 1. To acquire the semen samples, veterinary experts used a phase-contrast microscope and fixed the spermatozoa in glutaraldehyde. We define a model intracellular density distribution of the spermatozoid heads, according to the hypothesis of experts. Hence, the typical deviations from the model for normal distributions and non-normal distributions give a classification criterion. The goal is to automatically classify spermatozoid head images as normal or not-normal by means of their deviation from the model intracellular density distribution.

In Section 2, we present the methods we have used and the obtained results. Discussion and conclusions are given in Section 3

\section{Methods and Results}

\subsection{Pre-processing and Segmentation}

Boar sample images were captured by means of an optical phase-contrast microscope connected to a digital camera. The magnification used was $\times 40$ and the dimensions of each sample were $1600 \times 1200$ pixels. A boar sample image comprises a number of heads which can vary widely from one sample to the next (Fig. Th). Spermatozoid heads also present different orientations and tilts. After morphological processing to smooth the contours of the cells, they are isolated using segmentation by threshold applying Otsu's method. Finally, those regions that are smaller than an experimental obtained value of $45 \%$ of the average of the head area are removed as well as the heads next to the image boundaries (Fig. 1p). 


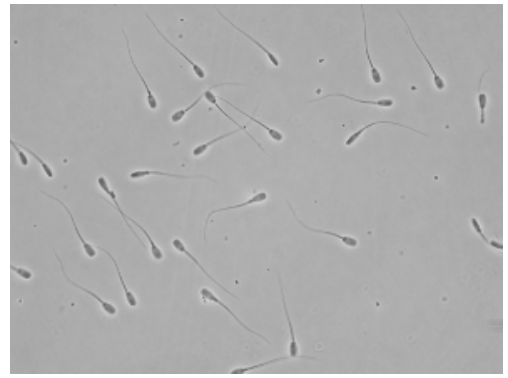

(a)

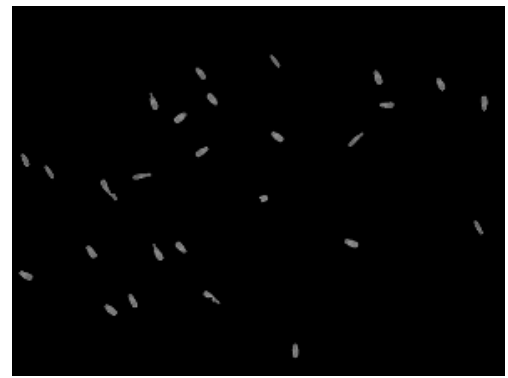

(b)

Fig. 1. (a) Boar semen sample image acquired using a phase-contrast microscope. (b) Image obtained after pre-processing and segmentation. Spermatozoid heads are grey-level distributions in oval shapes on a black background.

\subsection{Head Normalization}

A spermatozoid head presents an oval shape with an eccentricity between 1.75 and 2 . As heads in a sample have different orientations, we find the main axes of the ellipse that a head forms (Fig. 2a) to be able to rotate all the head images to the same horizontal orientation (Fig. 2b). Empirical measurements in head morphological analysis give a width from 4 to $5 \mu \mathrm{m}$ and a length from 7 to $10 \mu \mathrm{m}$. We re-scale all the head images to $19 \times 35$ pixels and consider a $2 \mathrm{D}$ function $f(x, y)$ defined by the grey levels of the image in the set of points which belongs to an ellipse whose major and minor axis are 35 and 19 respectively (Fig. 2 r).

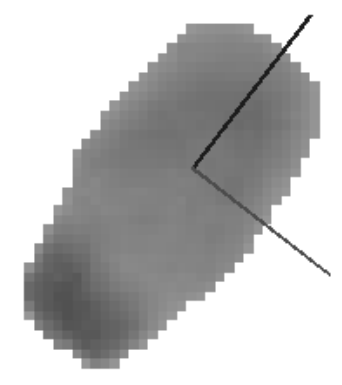

(a)

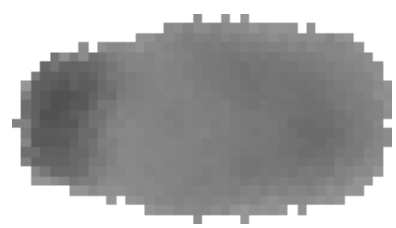

(b)

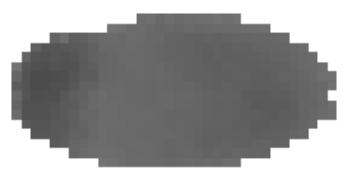

(c)

Fig. 2. (a) Spermatozoid head and main axes of the ellipse that it defines. (b) Rotated head. (c) After re-scaling and brightness and contrast normalization, 2D grey-level function obtained for the points of an ellipse with major and minor axis of 35 and 19 pixels, respectively.

Sample images differ in their brightness and contrast grey level. To normalise that, we develop a linear transformation of the 2D function to keep a determined mean and standard deviation (Fig. 2k). So, we define a new 2D function $g(x, y)$ as follows:

$$
g(x, y)=a f(x, y)+b,
$$




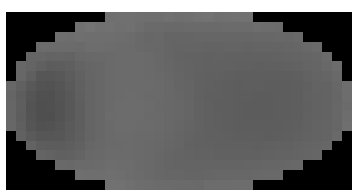

Fig. 3. Model of an intracellular distribution image considered as normal by veterinary experts, obtained from the average of a set of head images assumed as normal

where the coefficients $a$ and $b$ are computed as:

$$
a=\frac{\sigma_{g}}{\sigma_{f}}, \quad b=\mu_{g}-a \mu_{f} .
$$

The spermatozoid head images which experts consider as potentially normal take values for the mean and the standard deviation around 8 and 10 respectively. For this reason, we set $\sigma_{g}=8$ and $\mu_{g}=100$. The values $\mu_{f}$ and $\sigma_{f}$ correspond with the mean and the standard deviation of the function $f$, respectively.

\subsection{Model of a Normal Intracellular Distribution}

We describe a model density distribution as the average of a set of 34 heads assumed as normal by veterinary experts. Such heads have a grey-level intensity variation from left to right according to the dark post nucleus cap, the intermediate light area and a slightly darker part called acrosome that covers part of the cell nucleus. After the previous steps of pre-processing, segmentation and normalization, we compute a model 2D function as (Fig. 3):

$$
m(x, y)=\frac{1}{n} \sum_{i=1}^{n} g_{i}(x, y) .
$$

We also consider the standard deviation to assess the variability of the grey-levels for each point:

$$
\sigma(x, y)=\sqrt{\sum_{i=1}^{n} \frac{\left(g_{i}(x, y)-m_{i}(x, y)\right)^{2}}{n}}
$$

\subsection{Classification of Spermatozoid Head Images}

Apart from the set of images used to build the model, we employ two training sets of 44 head images labelled as "normal" (Fig. 4h) and 82 head images labelled as "nonnormal" (Fig. 4b) by veterinary experts according to the similarity to the intracellular distribution considered as potentially normal. For each image of those training sets, we apply the above mentioned stages and then compute a deviation from the model function using the $L_{\infty}$ norm:

$$
d=\max \left(\frac{|g(x, y)-m(x, y)|}{\sigma_{x y}}\right) .
$$




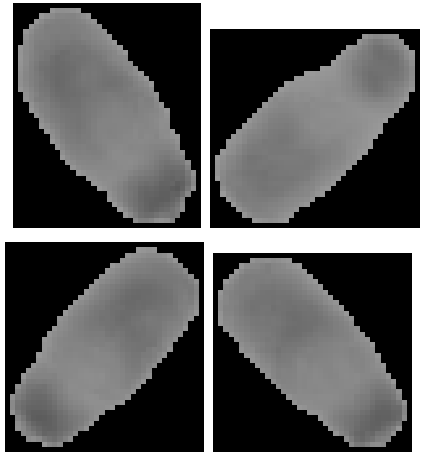

(a)
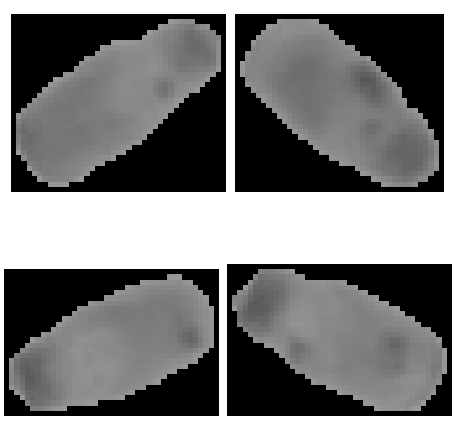

(b)

Fig. 4. Examples of heads that were classified by an expert as having an intracellular distribution that is (a) similar and (b) not similar to the assumed normal density distribution
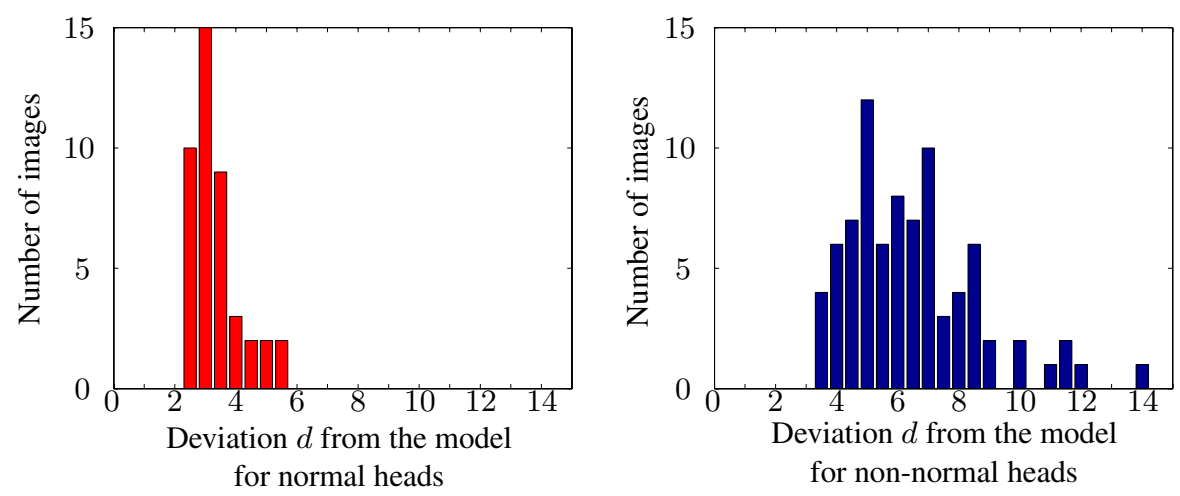

Fig. 5. Histograms of deviation from the model for (a) the set of head images considered as normal by the experts and (b) the set of spermatozoid head images that present intensity distributions not similar to the model

The obtained deviations for the 2D functions of the set of normal and non-normal head images yield two histograms, Fig. 5. In general, the deviation values obtained for normal heads are smaller than the ones obtained for non-normal ones. We can now classify heads by taking a certain value of the deviation as a decision criterion. If the deviation value obtained for a given head is below the decision criterion, that head is classified as normal, otherwise as non-normal. The two histograms overlap for values of the deviation between 3.5 and 5.5 and this means that it is not possible to achieve errorless classification by taking any value of the deviation as a decision criterion. If a high value of the decision criterion is taken (above 5.5), all normal heads will be classified as normal but a number of non-normal cells that have deviation values below 5.5 will be erroneously accepted as normal too. If a low decision criterion value (e.g. 3 ) is taken, all non-normal heads will be correctly rejected but a number of normal heads (with deviation values above 3) will be rejected as well. Fig. 6 shows the false acceptance and false rejection error as well as their sum as function of the value of the 


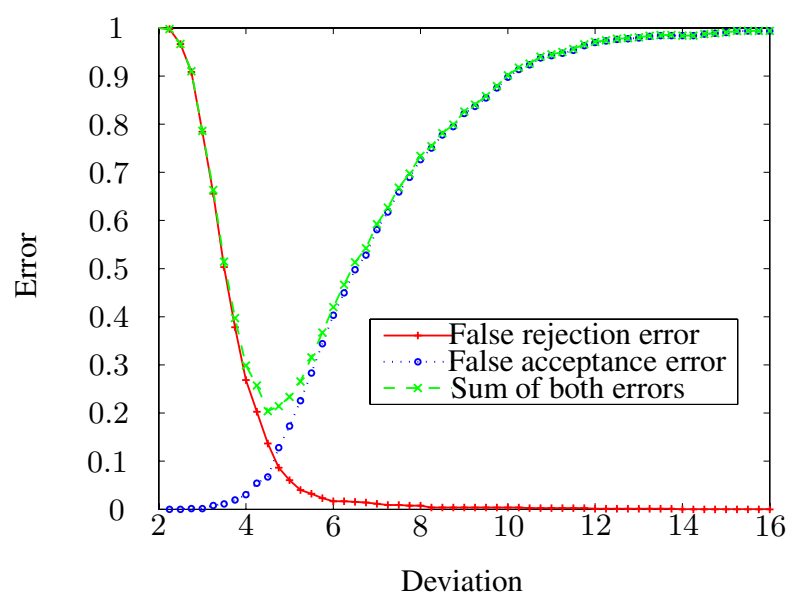

Fig. 6. Error rates in head classification obtained for the different values of the decision criterion. The red line shows the percentage of normal heads that are classified as non-normal (false rejection error); the blue line represents the fraction of non-normal heads misclassified (false acceptance error). The green line is the sum of both errors and it has a minimum for a decision criterion value of 4.25 .

decision criterion. The sum of the two errors has a minimum for the value 4.25 of the decision criterion and in the following we use this value for classification. We also used a Bayer classifier but it yielded error rates higher than the method explained previously.

\subsection{Experimental Results}

We use a test set of 1400 images of spermatozoid heads: 775 images of heads with a normal density distribution pattern according to veterinary experts and 625 images of heads which are not perceived as normal by the experts. We calculate the deviation of each such image from the model and classify it as normal if that deviation is less than 4.25. Otherwise the image is considered as non-normal. The false rejection error of normal heads is $13.68 \%$, and the false acceptance rate of non-normal heads is $6.72 \%$. The overall classification error is $20.40 \%$.

\section{Discussion and Conclusions}

We proposed a method to classify images of boar spermatozoid heads by means of their intracellular density distribution. A model of normal intensity distribution was defined using a set of head images that were assumed as potentially normal by veterinary experts. We used two training sets of images, one of normal and the other of non-normal heads, and computed the deviation of the grey-level intensity distribution of each such head image from the model distribution. That yields a histogram with the values of deviations of normal head distributions from the model and another histogram with the deviations of non-normal distributions from the model. These histograms were used to 
compute the value of a decision criterion in a two-class classification problem. Using this value of the decision criterion with a new test set of normal and non-normal head images, we obtained a global error of $20.40 \%$ with a false rejection error of normal heads of $13.68 \%$ and a false acceptance rate of non-normal heads of $6.72 \%$.

This result can not be compared with another works since there are no approaches which solve this problem considering the intracellular density distribution. Hence, in future works we will try to reduce this error using a more robust classification method. The obtained results will be tested in veterinary praxis using staining techniques to correlate it with sperm fertility.

\section{References}

1. Gravance, C., Garner, D., Pitt, C., Vishwanath, R., Sax-Gravance, S., Casey, P.: Replicate and technician variation associated with computer aided bull sperm head morphometry analysis (ASMA). International Journal of Andrology 22 (1999) 77-82

2. Hirai, M., Boersma, A., Hoeflich, A., Wolf, E., Foll, J., Aumuller, T., Braun, J.: Objectively measured sperm motility and sperm head morphometry in boars (Sus scrofa): relation to fertility and seminal plasma growth factors. J. Androl 22 (2001) 104-110

3. Wijchman, J., Wolf, B.D., Graafe, R., Arts, E.: Variation in semen parameters derived from computer-aided semen analysis, within donors and between donors. J. Androl. 22 (2001) 773-780

4. Suzuki, T., Shibahara, H., Tsunoda, H., Hirano, Y., Taneichi, A., Obara, H., Takamizawa, S., Sato, I.: Comparison of the sperm quality analyzer IIC variables with the computer-aided sperm analysis estimates. International Journal of Andrology 25 (2002) 49-54

5. Rijsselaere, T., Soom, A.V., Hoflack, G., Maes, D., de Kruif, A.: Automated sperm morphometry and morphology analysis of canine semen by the Hamilton-Thorne analyser. Theriogenology 62 (2004) 1292-1306

6. Verstegen, J., Iguer-Ouada, M., Onclin, K.: Computer assisted semen analyzers in andrology research and veterinary practice. Theriogenology 57 (2002) 149-179

7. Linneberg, C., Salamon, P., Svarer, C., Hansen, L.: Towards semen quality assessment using neural networks. In: Proc. IEEE Neural Networks for Signal Processing IV. (1994) 509-517

8. Garrett, C., Baker, H.: A new fully automated system for the morphometric analysis of human sperm heads. Fertil. Steril. 63 (1995) 1306-1317

9. Ostermeier, G., Sargeant, G., Yandell, T., Parrish, J.: Measurement of bovine sperm nuclear shape using Fourier harmonic amplitudes. J. Androl. 22 (2001) 584-594

10. Alegre, E., Sánchez, L., Aláiz, R., Dominguez-Fernández, J.: Utilización de momentos estadísticos y redes neuronales en la clasificación de cabezas de espermatozoides de verraco. In: XXV Jornadas de Automática. (2004)

11. Beletti, M., Costa, L., Viana, M.: A comparison of morphometric characteristics of sperm from fertile Bos taurus and Bos indicus bulls in Brazil. Animal Reproduction Science 85 (2005) 105-116 